\title{
Symptomatic Profiles of Patients With Polycythemia Vera: Implications of Inadequately Controlled Disease
}

Holly Geyer, Robyn Scherber, Heidi Kosiorek, Amylou C. Dueck, Jean-Jacques Kiladjian, Zhijian Xiao, Stefanie Slot, Sonja Zweegman, Federico Sackmann, Ana Kerguelen Fuentes, Dolores Hernández-Maraver, Konstanze Döhner, Claire N. Harrison, Deepti Radia, Pablo Muxi, Carlos Besses, Francisco Cervantes, Peter L. Johansson, Bjorn Andreasson, Alessandro Rambaldi, Tiziano Barbui, Karin Bonatz, Andreas Reiter, Francoise Boyer, Gabriel Etienne, Jean-Christophe Ianotto, Dana Ranta, Lydia Roy, Jean-Yves Cahn, Norman Maldonado, Giovanni Barosi, Maria L. Ferrari, Robert Peter Gale, Gunnar Birgegard, Zefeng Xu, Yue Zhang, Xiujuan Sun, Junqing Xu, Peihong Zhang, Peter A.W. te Boekhorst, Suzan Commandeur, Harry Schouten, Heike L. Pahl, Martin Griesshammer, Frank Stegelmann, Thomas Lehmann, Zhenya Senyak, Alessandro M. Vannucchi, Francesco Passamonti, Jan Samuelsson, and Ruben A. Mesa

Listen to the podcast by Dr Stein at www.jco.org/podcasts

Author affiliations appear at the end of this article.

Published online ahead of print at www.jco.org on November 23, 2015.

Presented in poster format at the 56th American Society of Hematology Conference, San Francisco, CA, December 6-9, 2014

Authors' disclosures of potential conflicts of interest are found in the article online at www.jco.org. Author contributions are found at the end of this article.

Corresponding author: Ruben A. Mesa, MD, Division of Hematology and Oncology, Mayo Clinic, 13400 E Shea Blvd, Scottsdale, AZ 85259; e-mail: mesa.ruben@mayo.edu.

(C) 2015 by American Society of Clinical Oncology

0732-183X/16/3402w-151w/\$20.00 DOI: 10.1200/JCO.2015.62.9337

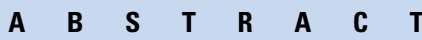

\section{Purpose}

Polycythemia vera (PV) is a myeloproliferative neoplasm (MPN) associated with disabling symptoms and a heightened risk of life-threatening complications. Recent studies have demonstrated the effectiveness of $J A K$ inhibitor therapy in patients with PV patients who have a history of prior hydroxyurea (HU) use (including resistance or intolerance), phlebotomy requirements, and palpable splenomegaly. We aimed to determine how these features contribute alone and in aggregate to the PV symptom burden.

\section{Patients and Methods}

Through prospective evaluation of 1,334 patients with PV who had characterized symptom burden, we assessed patient demographics, laboratory data, and the presence of splenomegaly by disease feature (ie, known HU use, known phlebotomy requirements, splenomegaly).

\section{Results}

The presence of each feature in itself is associated with a moderately high symptom burden (MPN symptom assessment form [SAF] total symptom score [TSS] range, 27.7 to 29.2) that persists independent of PV risk category. In addition, symptoms incrementally increase in severity with the addition of other features. Patients with PV who had all three features (PV-HUPS) faced the highest total score (MPN-SAF TSS, 32.5) but had similar individual symptom scores to patients with known $\mathrm{HU}$ use (PV-HU), known phlebotomy (PV-P), and splenomegaly (PV-S).

\section{Conclusion}

The results of this study suggest that patients with PV who have any one of the features in question (known HU use, known phlebotomy, or splenomegaly) have significant PV-associated symptoms. Furthermore, it demonstrates that many PV symptoms remain severe independent of the number of features present.

\section{J Clin Oncol 34:151-159. (C) 2015 by American Society of Clinical Oncology}

\section{INTRODUCTION}

Polycythemia vera (PV) is a myeloproliferative neoplasm (MPN) that evolves from dysregulated clonal erythropoiesis. The condition is recognized for its distinct molecular profile (JAKV617F), burdensome symptoms, predilection for thrombosis, and capacity to transform into acute myelogenous leukemia (AML) or myelofibrosis (MF). Treatments are primarily directed at preventing life-threatening complications (eg, thrombosis), and the choice of interventions is typically based on risk assessment. ${ }^{1,2}$ Despite many treatment advancements, studies continue to demonstrate that the PV symptom burden remains undermanaged. ${ }^{3}$ In a 2007 internet survey of 405 patients with PV who were receiving standard treatment, the authors found that symptoms-including fatigue (85\%), pruritus (65\%), night sweats (49\%), bone pain $(43 \%)$, fevers $(13 \%)$, and undesired weight loss $(10 \%)$ - directly contributed to poor overall quality of life and compromised functionality. ${ }^{3}$ Palpable splenomegaly, present in up to 
$36 \%$ of patients with PV, is especially bothersome and contributes to other symptoms and complications, including early satiety, bloating, pain, portal hypertension, weight loss, and exacerbation of cytopenias. $^{4-6}$

A variety of therapies have been used in PV, most of which aim to reduce thrombotic risk, because inadequately controlled hematocrit levels have resulted in higher rates of cardiovascular events $(10.9 \% v 4.4 \%)$ and death $(4.4 v 1.1$ per 100 person-years $)$ and have perpetuated symptoms such as headaches and coughing. ${ }^{7}$ The CYTO-PV study clearly demonstrated improved thrombotic outcomes with maintenance of a hematocrit less than $45 \%$ through phlebotomy. ${ }^{8}$ However, recent studies have shown that strict control of hematocrit levels also may be associated with a higher symptom burden, including worsened fatigue, pruritus, concentration issues, insomnia, weight loss, and night sweats. ${ }^{7}$ Hydroxyurea (HU) remains first-line treatment for patients with PV who need cytoreductive therapy. ${ }^{9}$ However, resistance and intolerance to HU has been reported in $11 \%$ and $13 \%$ of patients, respectively, and, furthermore, may contribute to a 5.6-fold increased risk of death. ${ }^{10,11}$

More recently, attentions have turned to the development of treatments that specifically address PV symptoms. The phase III prospective Randomized Study of Efficacy and Safety in Polycythemia Vera with JAK Inhibitor INCB018424 versus Best Supportive Care (RESPONSE) evaluated the JAK2 inhibitor ruxolitinib against best available therapy (BAT) in patients with PV who had the following features: resistance or intolerance to $\mathrm{HU}$; baseline splenomegaly $\left(>450 \mathrm{~cm}^{3}\right)$; and phlebotomy dependence. ${ }^{12}$ The study demonstrated that patients with PV who had all three features suffered from a significant symptom burden and that ruxolitinib effectively alleviated these symptoms. As a result, on December 4, 2014, the FDA approved ruxolitinib for a select subset of the PV population: patients resistant or intolerant to HU.

This tremendous advancement for the PV population is limited by the issue of the restricted availability of ruxolitinib to select PV patients whose baseline symptomatology has not yet been fully characterized or compared to other PV patients who lack HU resistance/intolerance but still potentially face high symptom burdens. In this study, we sought to compare and contrast the baseline symptoms of patients with PV who have known HU use (PV-HU), known phlebotomy (PV-P), palpable splenomegaly (PV-S), or all three features (PV-HUPS).

\section{PATIENTS AND METHODS}

\section{Survey Development and Collection}

PV patients were recruited from international academic, private, and government medical institutions during routine office visits in a format previously published for validation of the MPN symptom assessment form (MPN-SAF) ${ }^{13}$ and through an online international survey (MPN fatigue project). ${ }^{14}$ In this study, anemia, thrombocytopenia, and leukopenia were defined as hemoglobin less than $11 \mathrm{~g} / \mathrm{dL}$, platelet count less than $150 \times 10^{9}$ cells/L, and white blood cell count less than $3.5 \times 10^{9}$ cells/L, respectively. Data were collected in English, Chinese, Dutch, French, German, Italian, Spanish, and Swedish languages.

\section{Symptom Assessment}

Assessment of MPN symptoms was performed by using the validated MPN-specific patient-reported outcome tool, the MPN-SAF total symptom score (MPN-SAF TSS; MPN-10). ${ }^{2}$ Assessed symptoms were fatigue, early satiety, abdominal discomfort, inactivity, concentration problems, night sweats, itching, bone pain, fevers, and weight loss. All items were evaluated on a 0 (absent) to 10 (worst imaginable) scale. For individuals who completed at least six of the 10 MPN-SAF TSS items, the survey was scored by multiplying the average score across items by 10 to achieve a 0-to-100 scaled score. The survey was collected in the aforementioned languages, and original language validations were conducted with a patient-reported outcome translation method.

\section{Clinical Feature Subgroup Assignment}

Clinical feature subgroups of PV were grouped according to the type and number of features present. Features were defined as known HU use, known phlebotomy, and splenomegaly. Subgroups consisted of those with no features or with one, two, or all three features present. Patient demographic and clinical variables, MPN-10 TSS score, and individual MPN-10 TSS items were compared among feature subgroups. In the MPN database, both current and prior use of HU/phlebotomy were assessed as separate questions. In the fatigue survey, however, only use of these therapies was assessed, and current versus previous use was not distinguished. Patients with a history of prior splenectomy were excluded. Control groups for each PV feature were derived from the remaining total PV patient cohort that lacked the specified feature (PV-cHU, PV-cP, PV-cS, PV-cHUPS); patients in whom the trait status was unknown were excluded from each respective control group.

\section{Prognostic Scoring}

Prognostic scoring for PV survival was calculated with the Leukemia 2013 prognostic scoring model developed by Tefferi et al. ${ }^{6}$ This scoring system includes the variables of age 67 years or older ( 5 points), age 57 to 66 years $\left(2\right.$ points), WBC $15 \times 10^{9} / \mathrm{L}$ or greater ( 1 point), and prior thrombosis ( 1 point) to risk stratify patients into high risk ( $\geq 3$ points), intermediate risk ( 1 to 2 points), or low risk (0 points).

\section{MPN Symptom Burden Severity Assessment}

Determination of clinically meaningful difference in symptom severity for patients with PV was based on the 2013 MPN symptom burden response thresholds criteria. ${ }^{15}$ With this criteria, the TSS for each patient was analyzed to place the patient into the quartiles of low symptom burden (TSS, zero to seven), intermediate symptom burden (TSS, eight to 17), moderately high symptom burden (TSS, 18 to 31 ), or high symptom burden (TSS, $\geq 32$ ).

\section{Statistical Analysis}

Continuous variables were compared with an analysis of variance, and dichotomous data were compared with the $\chi^{2}$ test. Overall MPN-10 TSS score and individual symptoms were compared among various subgroups with the $t$ test. Comparison of symptoms among groups used $t$ tests. Statistical significance was set to $P<.05$. SAS version 9.3 (Cary, NC) was used for analysis.

\section{RESULTS}

\section{Demographics}

A total of 1,334 patients were included for analysis and had the following characteristics: known $\mathrm{HU}$ use (PV-HU, $\mathrm{n}=499)$, known phlebotomy (PV-P, $\mathrm{n}=646$ ), palpable splenomegaly (PV-S, $\mathrm{n}=369$ ), or all three features (PV-HUPS, $\mathrm{n}=148$; Table 1). Included patients were recruited from the MPN-10 TSS validation study $(\mathrm{n}=717)$ and the 2014 FATIGUE study $(\mathrm{n}=617)$. Patients on the MPN-10 TSS validation study were slightly older than those 


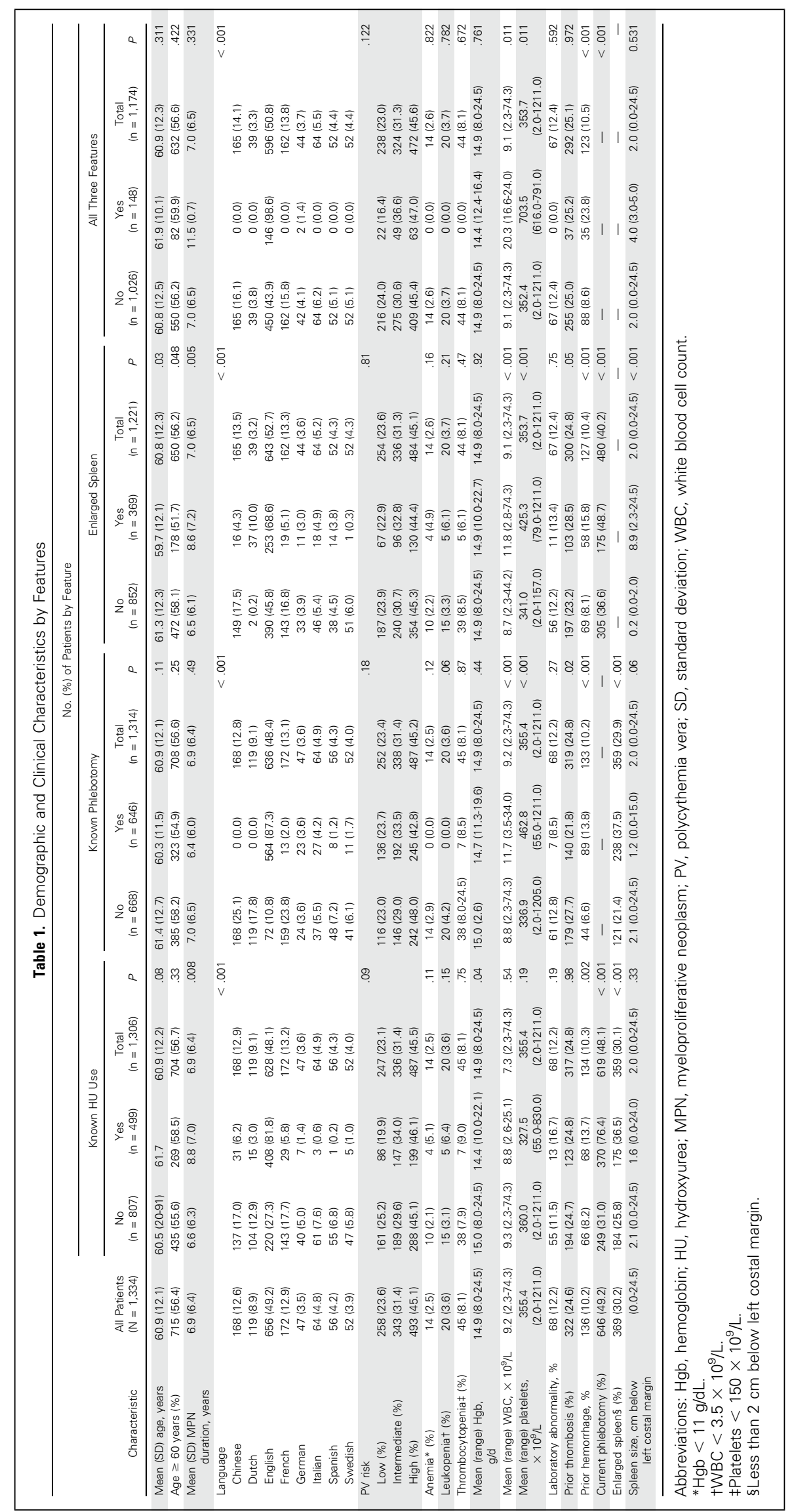


on the fatigue survey (mean [deviation $\{\mathrm{SD}\}$ ], 61.8 [12.7] years $v 59.7$ [11.3] years; $P=.003$ ), and a higher proportion of patients on the validation study than on the fatigue survey had high-risk scores $(49.2 \%$ $v 40.8 \% ; P=.017)$. In addition, the MPN-10 TSS database included an international cohort of patients, and the fatigue survey was completed only by patients from the United States. Each PV subgroup (PV-HU, PV-P, PV-S, and PV-HUPS) was compared with a control group composed of the remaining total PV population that lacked the trait, including those without a history of known HU use (PV-cHU, $\mathrm{n}=$ 807), without known phlebotomy (PV-cP, $\mathrm{n}=668$ ), without splenomegaly (PV-cS, $\mathrm{n}=852$ ), or without all three features (PV-cHUPS, $\mathrm{n}=1,026)$. Patient ages were similar among subgroups (PV-HU, 61.7 years; PV-S, 59.7 years; PV-P, 60.3 years; PV-HUPS, 61.9 years) and their respective comparison groups (range, 60.8- to 61.3 years).

\section{Clinical Variables}

Disease duration varied among PV-HU, PV-P, PV-S, and PVHUPS subgroups (range, 6.5 to 11.5 years; Table 1). Patients in both the PV-HU and PV-S subgroups demonstrated significantly longer disease durations than those patients in the respective control groups that lacked the descriptive feature (PV-HU, 8.8 years $v$ PV-cHU, 6.6 years $[P=.008]$; PV-S, 8.6 years $v$ PV-cS, 6.5 years $[P=.005])$. No statistical differences in PV risk scores were noted among any of the subgroups or their respective comparison groups, and most patients met high-risk criteria (range, $42.8 \%$ to $47.0 \%$ ). The prevalence of prior thrombosis among subgroups ranged from $21.8 \%$ to $28.5 \%$, and a statistical difference was noted in the prevalence of PV-P and PV-S in relation to their control groups (PV-P, 21.8\% $v$ PV-cP, 27.7\% $[P=.02]$; PV-S, 28.5\% $v$ PV-cS, 23.2\% [P=.05]). History of prior hemorrhage and splenomegaly were statistically more prominent in all subgroups when compared to their respective comparison groups.

\section{Laboratory Abnormalities}

The presence of laboratory abnormalities varied among subgroups (Table 1). For patients in the PV-HU, PV-P, and PV-S groups, rates of anemia (range, $0 \%$ to $5.1 \%$ ) leukopenia (range, $0 \%$ to $6.4 \%$ ), and thrombocytopenia (range, $0 \%$ to $9.0 \%$ ) were low and were without statistical difference when compared with their respective control groups. Mean hemoglobin levels were similar among patients in the PV-HU, PV-P, and PV-S subgroups (range, 14.4 to $14.9 \mathrm{~g} / \mathrm{dL}$ ). Patients in the PV-HUPS subgroup had the highest levels of leukocytosis and thrombocytosis among feature groups. Wide variability was noted in the mean WBC and platelet counts between subgroups; counts in patients with PV-HUPS $\left(20.3 \times 10^{9} / \mathrm{L}\right.$ and $703.5 \times 10^{9} / \mathrm{L}$, respectively $)$ were markedly higher than those in the PV-HU $\left(8.8 \times 10^{9} / \mathrm{L}\right.$ and $327.5 \times 10^{9} / \mathrm{L}$, respectively), PV-P $\left(11.7 \times 10^{9} / \mathrm{L}\right.$ and $462.8 \times 10^{9} / \mathrm{L}$, respectively $)$, or PV-S $\left(11.8 \times 10^{9} / \mathrm{L}\right.$ and $425.3 \times 10^{9} / \mathrm{L}$, respectively) subgroups. Statistical differences in WBC and platelet counts were also noted between patients when comparing PV-P, PV-S, or PV-HUPS subgroups and their respective control groups.

\section{High Symptom Burden in Individual Feature Subgroups}

Intermediate to high symptom burdens were noted in all feature subgroups, and the highest symptom burden was expressed by patients in the PV-HUPS subgroup (TSS, 32.5; range, 27.7 to 32.5; Figs 1 and 2). Patients in the PV-HUPS subgroup also had higher individual scores for all symptoms assessed, with the exception of fever. However, numerous scores for individual symptoms, including abdominal discomfort, inactivity, night sweats, bone pain, and weight loss, were similarly high between PV-HUPS and other single-feature PV subgroups. MPN-10 total symptom scores were similar between PV-HU (29.2), PV-P (27.7), and PV-S (28.8) subgroups. Patients in the PV-HU subgroup had the highest mean symptom scores for microvascular and cytokine-related complaints, including fatigue, inactivity, concentration problems, night sweats, and bone pain. Patients in the PV-P subgroup had the lowest mean symptoms scores for items related to abdominal complaints, including early satiety, abdominal discomfort, and weight loss. They also had the lowest overall score for pruritus. Patients in the PV-S subgroup were most likely to have abdominalrelated complaints, including early satiety and weight loss, and were least likely to have fatigue. When each feature subgroup was compared with their respective control group, the TSS and most of the individual symptoms were higher in patients who possessed the feature in comparison to those who lacked it (Figs 1 and 2).

\section{Worsening Symptom Burden With Increasing Number of Features}

Data also were compared between PV cohorts on the basis of the number of features present within each patient (Table 2). A total of 362 patients with PV had zero features; 348 had one feature (PV-HU, $\mathrm{n}=$ 82; PV-P, $\mathrm{n}=165$; PV-S, $\mathrm{n}=101$ ); 315 had two features (PV-HU + PV$\mathrm{P}, \mathrm{n}=215 ; \mathrm{PV}-\mathrm{P}+\mathrm{PV}-\mathrm{S}, \mathrm{n}=80 ; \mathrm{PV}-\mathrm{HU}+\mathrm{PV}-\mathrm{S}, \mathrm{n}=20) ; 617$ had one or two features; and 148 had all three features (PV-HUPS).

Mean age statistically differed among patients by the number of features they possessed $(P=.002)$. Patients who had zero features were slightly older (62.5 years) than those with one to two features (59.8 years). Patients who had all three features had the highest mean WBC count $\left(20.3 \times 10^{9} / \mathrm{L} v 8.5\right.$ to $\left.10.0 \times 10^{9} / \mathrm{L} ; P=.001\right)$, highest platelet levels $\left(703.5 \times 10^{9} / \mathrm{L} v 332.8\right.$ to $\left.384.7 \times 10^{9} / \mathrm{L} ; P<.001\right)$, and highest history of prior hemorrhage $(23.8 \% v 4.7 \%$ to $10.7 \%$; $P<.001)$. No differences were noted in PV risk scores; the presence of anemia, leukopenia, or thrombocytopenia; mean hemoglobin level; or history of prior thrombosis.

When symptom burdens were compared, scores for all individual questions statistically differed by the number of features present, with the exception of fever and weight loss (Figs 3 and 4). Patients who had one feature had higher total and individual symptom scores than patients who had zero features. Similarly, patients who had two features had higher individual and total symptom scores than patients who had one feature (PV-P + other $v$ PV-P, $P=.05$; PV-HU + other $v$ PV-HU, $P=.01$ ), and patients who had all three features (PV-HUPS) had higher individual and total symptom scores (all $P<.01$ ) than patients who had one or two features. Overall, symptom scores were highest and most prevalent for the item of fatigue followed by microvascular-related symptoms, including concentration difficulties and pruritus.

\section{DISCUSSION}

The results of this study clearly demonstrate that the symptom burden in patients with PV is substantial, independent of whether a patient has used $\mathrm{HU}$, has received phlebotomy, or has splenomegaly. 


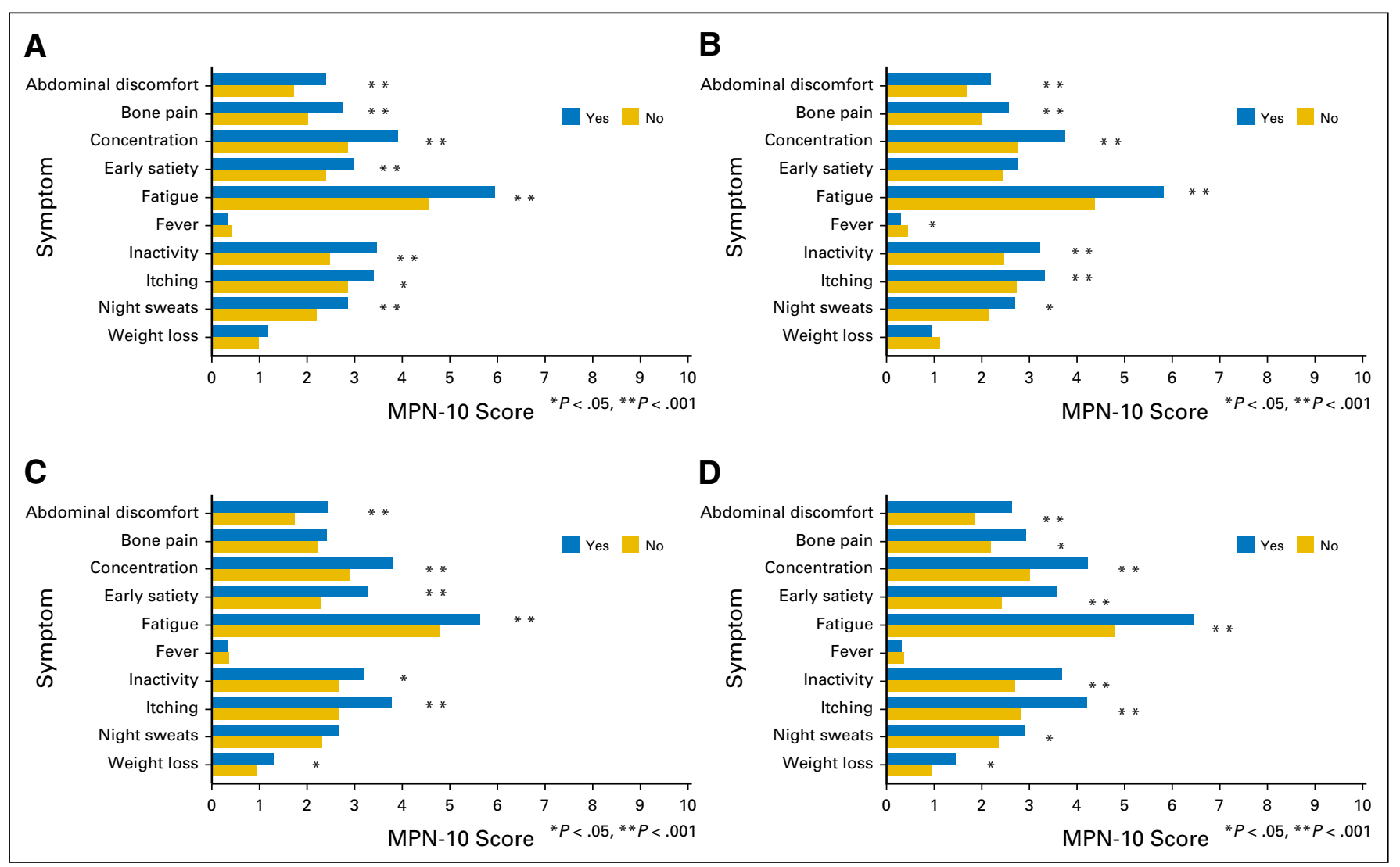

Fig 1. Myeloproliferative neoplasm symptom assessment form (MPN-10) with individual symptoms by features. Mean symptom scores by (A) known hydroxyurea use, (B) known phlebotomy, (C) enlarged spleen, or (D) all three features.

With the 2013 symptom response thresholds criteria, ${ }^{15}$ patients with PV who had all three features (PV-HUPS) met criteria for symptom quartile 4 (TSS, 32.5; high symptom burden profile). To put this into perspective, the average TSS of patients with myelofibrosis falls within symptom quartile 3 (TSS, 25.3), which suggests that disease burden is exceptionally high within this PV population. ${ }^{2}$ Importantly, all patients with PV who had only one feature (PV-P, PV-S, or PV-HU) also ranked within symptom quartile 3 (moderately high symptom burden profile), which demonstrates suboptimal symptom control. Patients with PV who had any given feature were statistically more symptomatic by MPN-10 TSS than their control group that lacked the individual feature, which suggests that the presence of splenomegaly, HU use, or phlebotomy requirement is independently associated with a substantial symptom burden.

This study also identified an additive relationship between the number of features present and the degree of symptoms expressed. This is especially true for participants in the PV-P and PV-HU subgroups. For all categories, patients who possessed two features were significantly more symptomatic than those who had one feature. Similarly, those who had three features had more symptoms than those who had one or two features. This finding corresponds with the results in Table 2 that demonstrate a statistical difference in risk scores on the basis of the number of features present. Positive associations were also noted between the severity of thrombocythemia, leukocytosis, or prior hemorrhage and the presence of multiple features, as demonstrated by patients in the PV-HUPS subgroup.

It is notable that many scores for individual symptoms in patients with PV-HUPS were similar to those of patients in the single-feature PV-HU, PV-P, and PV-S subgroups (Fig 1). This is especially true for cytokine-driven items (night sweats, bone pain, weight loss) and abdominal-related complaints (abdominal discomfort, weight loss) in patients in the PV-HU subgroup. Of interest, the TSS of patients in the PV-HU subgroup was similar to that of patients in the PV-P and PV-S subgroups (29.2 $v 27.7$ to 28.8). Historically, HU has been reserved for high-risk PV. Given that patients in the PV-P and PV-S subgroups did not statistically differ by risk category compared with their respectively control groups, the higher symptom score of patients in the PV-P and PV-S subgroups implies that high symptomatic burden exists independent of risk category and, furthermore, that symptoms remain undermanaged in low-risk patients with PV who are not deemed candidates for cytoreductive therapy. Although still meeting criteria for moderately high symptom burden, PV patients with a history of phlebotomy had a slightly lower symptom burden than patients in the other PV cohorts for both total score and individual items. Patients in the PV-P group did not differ from those in their respective control groups by risk category, so it is possible that phlebotomy in itself has symptom-ameliorating characteristics that have yet to be elucidated.

It is also worth noting that the items of weight loss and fevers were least likely to differ between feature groups and their 


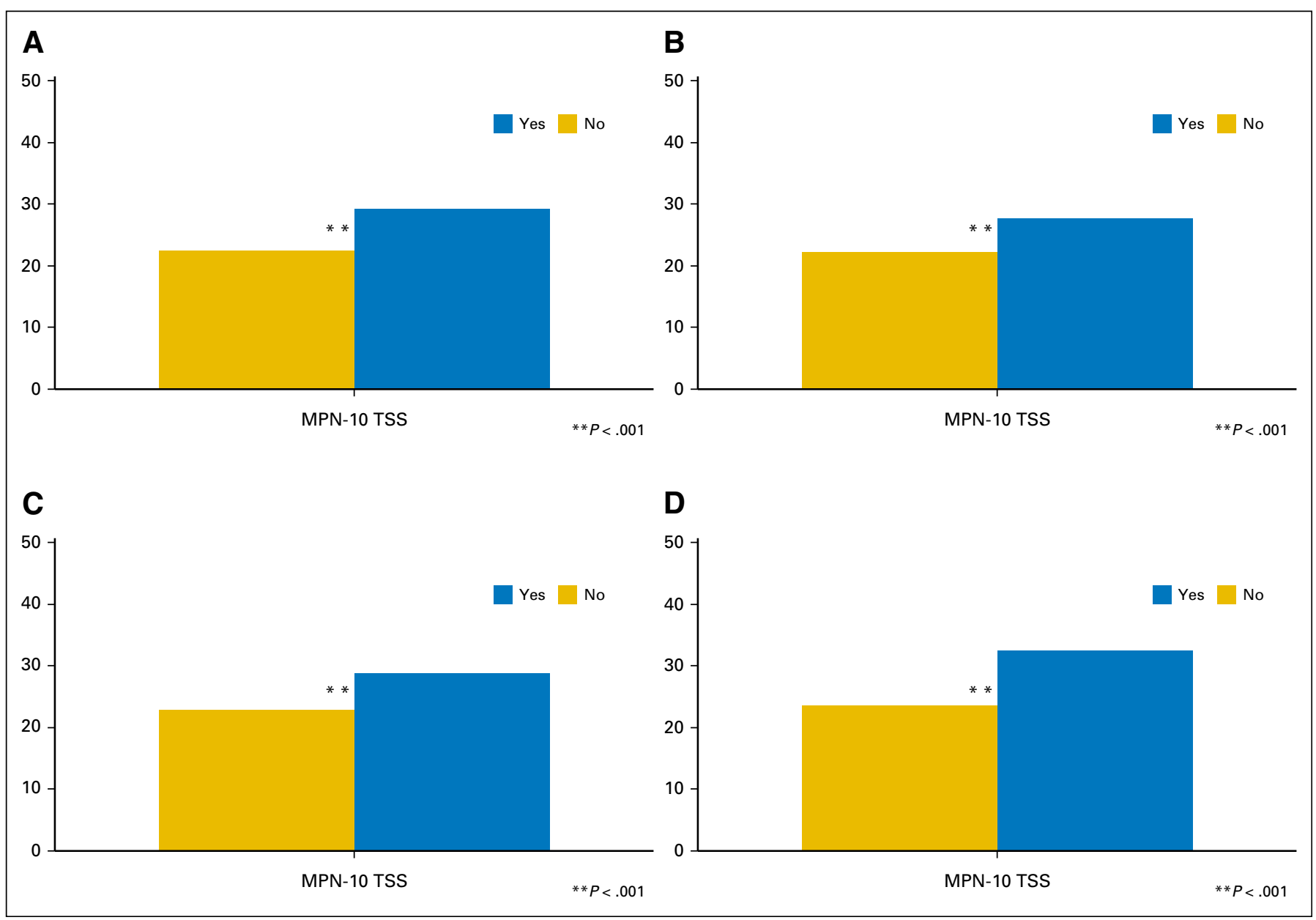

Fig 2. Myeloproliferative neoplasm symptom assessment form (MPN-10) total symptom score (TSS) by features. Mean MPN-10 TSS by (A) known hydroxyurea use, (B) known phlebotomy, (C) enlarged spleen, or (D) all three features.

associated controls or to vary by the number of features possessed. This observation implies that these disease features are not altered by the interventions (phlebotomy, HU) or the presence of splenomegaly. It is possible that the unique neurohormonal and cytokine environment (interleukin 4 [IL-4], fibroblast growth factor b [FGF-b], hepatocyte growth factor [HGF], and RANTES) induced by PV results in hypothalamic sensitization that contributes to weight loss and fever development. ${ }^{16}$ Alternatively, it is also possible that these are the two easiest symptoms to externally validate with objective information, directing the patient away from subjective reporting. It is worth noting that patients in both the PV-S and PV-HU subgroups differed in disease length from their respective control groups. However, the compared cohorts did not differ by risk scores, which suggests that disease length had a minimal impact on disease burden.

It is important to emphasize that there are limitations in making direct comparisons between patients in this study and the population represented in the RESPONSE trial. ${ }^{12}$ First, this study did not differentiate whether therapies (HU/phlebotomy) were being provided currently or had been used remotely. The RESPONSE trial specifically included patients with resistance or intolerance to $\mathrm{HU}$ (which suggested that patients were no longer receiving therapy) and phlebotomy dependence, defined as two or more phlebotomies within 24 weeks prior to screening and at least one within 16 weeks before screening. It is plausible that patients in whom $\mathrm{HU}$ has failed and/or patients who have current phlebotomy requirements may face a different symptom burden than that of patients who have a different timeline of therapy use. However, our population was a mixture of patients with current and prior therapy use, so we feel that the differences would likely be minimal. Furthermore, patients with resistance or intolerance to $\mathrm{HU}$ would be likely to express more symptoms, not less, which additionally substantiates our concerns that this population remains symptomatically undermanaged. It also is worth noting that our patient sample was composed of two different cohorts acquired from separate databases (the MPN-10 TSS validation survey and the FATIGUE survey). Participation in the survey for both groups was voluntary, and neither population received compensation for involvement. As such, we believe that selection bias was minimal but not directly investigated within this study.

The results of this investigation plainly show that symptoms in patients with PV who have known HU use, known phlebotomy requirements, and splenomegaly are greater than in those patients who lack these features. Furthermore, there is an additive effect on symptom burden as more features are included in a patient's profile. As discussed, the symptom burdens of patients with PV who have splenomegaly and/or phlebotomy requirements remain 


\begin{tabular}{|c|c|c|c|c|c|}
\hline \multirow[b]{2}{*}{ Characteristic } & \multicolumn{3}{|c|}{ No. $(\%)$ of Patients by Number of Features } & \multirow{2}{*}{$\begin{array}{l}\text { All Patients } \\
(\mathrm{N}=1,116)\end{array}$} & \multirow[b]{2}{*}{$P$} \\
\hline & $0(n=362)$ & $1-2(n=617)$ & $3(n=137)$ & & \\
\hline Mean (SD) age, years & $62.5(12.8)$ & $59.8(12.3)$ & $61.9(10.1)$ & 60.9 (12.3) & .002 \\
\hline Age $\geq 60$ years & $224(61.9)$ & $326(52.8)$ & $82(59.9)$ & $632(56.6)$ & .02 \\
\hline Mean (SD) PV duration, years & $6.6(6.2)$ & $7.4(6.8)$ & $11.5(0.7)$ & $7.0(6.5)$ & .30 \\
\hline PV risk & & & & & .04 \\
\hline Low & $75(22.0)$ & $141(25.2)$ & $22(16.4)$ & $238(23.0)$ & \\
\hline Intermediate & $94(27.6)$ & $181(32.4)$ & 49 (36.6) & $324(31.3)$ & \\
\hline High & $172(50.4)$ & $237(42.4)$ & $63(47.0)$ & $472(45.6)$ & \\
\hline Anemia* & $7(2.1)$ & $7(3.4)$ & $0(0.0)$ & $14(2.6)$ & .62 \\
\hline Leukopenia† & $13(3.9)$ & $7(3.4)$ & $0(0.0)$ & $20(3.7)$ & .93 \\
\hline Thrombocytopeniał & $29(8.6)$ & $15(7.4)$ & $0(0.0)$ & $44(8.1)$ & .80 \\
\hline Mean (SD) Hgb, g/dL & $15.0(2.7)$ & $14.8(2.3)$ & $14.4(2.8)$ & $14.9(2.5)$ & .42 \\
\hline Mean (SD) WBC, $\times 10^{9} / \mathrm{L}$ & $8.5(5.5)$ & $10.0(7.4)$ & $20.3(5.2)$ & $9.1(6.4)$ & .001 \\
\hline Mean (SD) platelets, $\times 10^{9} / \mathrm{L}$ & $332.8(174.8)$ & $384.7(226.1)$ & $703.5(123.7)$ & $353.7(198.0)$ & $<.001$ \\
\hline Laboratory abnormality & $43(12.8)$ & 24 (11.8) & $0(0.0)$ & 67 (12.4) & .82 \\
\hline Prior thrombosis & $95(26.2)$ & $160(24.4)$ & $37(25.2)$ & $292(25.1)$ & .83 \\
\hline Prior hemorrhage & $17(4.7)$ & $71(10.7)$ & $35(23.8)$ & $123(10.5)$ & $<.001$ \\
\hline
\end{tabular}

unacceptably high and rival those of patients who meet high-risk criteria and who, therefore, become candidates for HU treatment. These findings are far-reaching because emerging therapies offer the potential to reduce phlebotomy requirements, protect against vascular events, improve the symptomatic burden, and potentially offer protection against disease transformation. Recent studies to characterize the symptoms inherent to phlebotomy ${ }^{7}$ and $\mathrm{HU}$ use $^{10,11}$ have made the philosophy of individualized PV treatments according to symptom response an increasingly appealing approach. ${ }^{17}$ As demonstrated in the RESPONSE trial, ruxolitinib improves symptoms and controls hematocrit in patients who have the combined three features. ${ }^{12}$ The results of this study suggest that patients with PV who have even one or two features may benefit from JAK2 inhibitor treatment as well. Other novel therapies, including histone deacetylase (HDAC) inhibitors and heat-shock protein-90 (HSP-90) inhibitors, are under active investigation in $\mathrm{PV}$, and results are anxiously anticipated.

\section{AUTHORS' DISCLOSURES OF POTENTIAL CONFLICTS OF INTEREST}

Disclosures provided by the authors are available with this article at www.jco.org

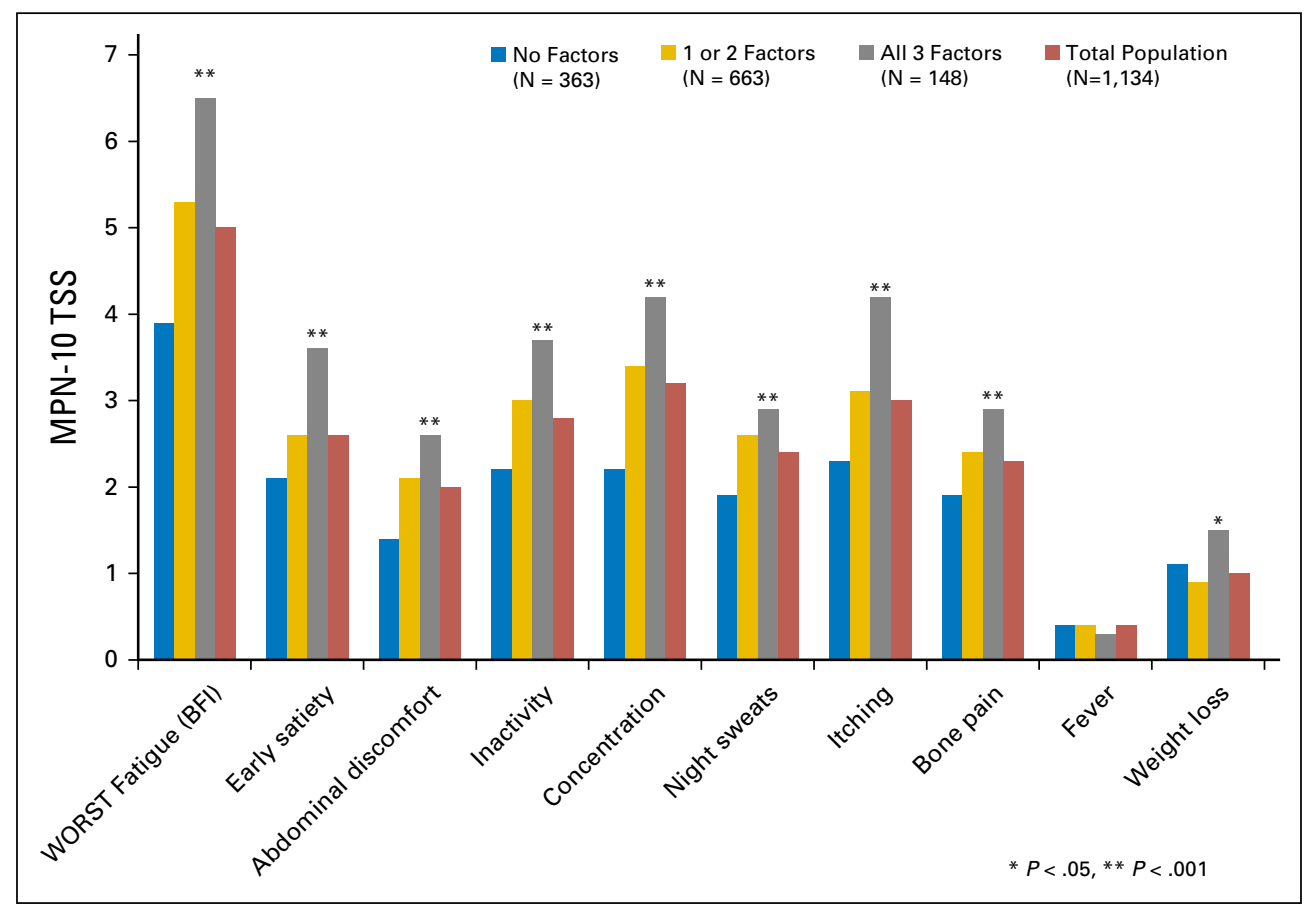

Fig 3. Myeloproliferative neoplasm symptom assessment form (MPN-10) total symptom score (TSS) symptoms according to the number of features present. BFI, Brief fatigue inventory scale. 


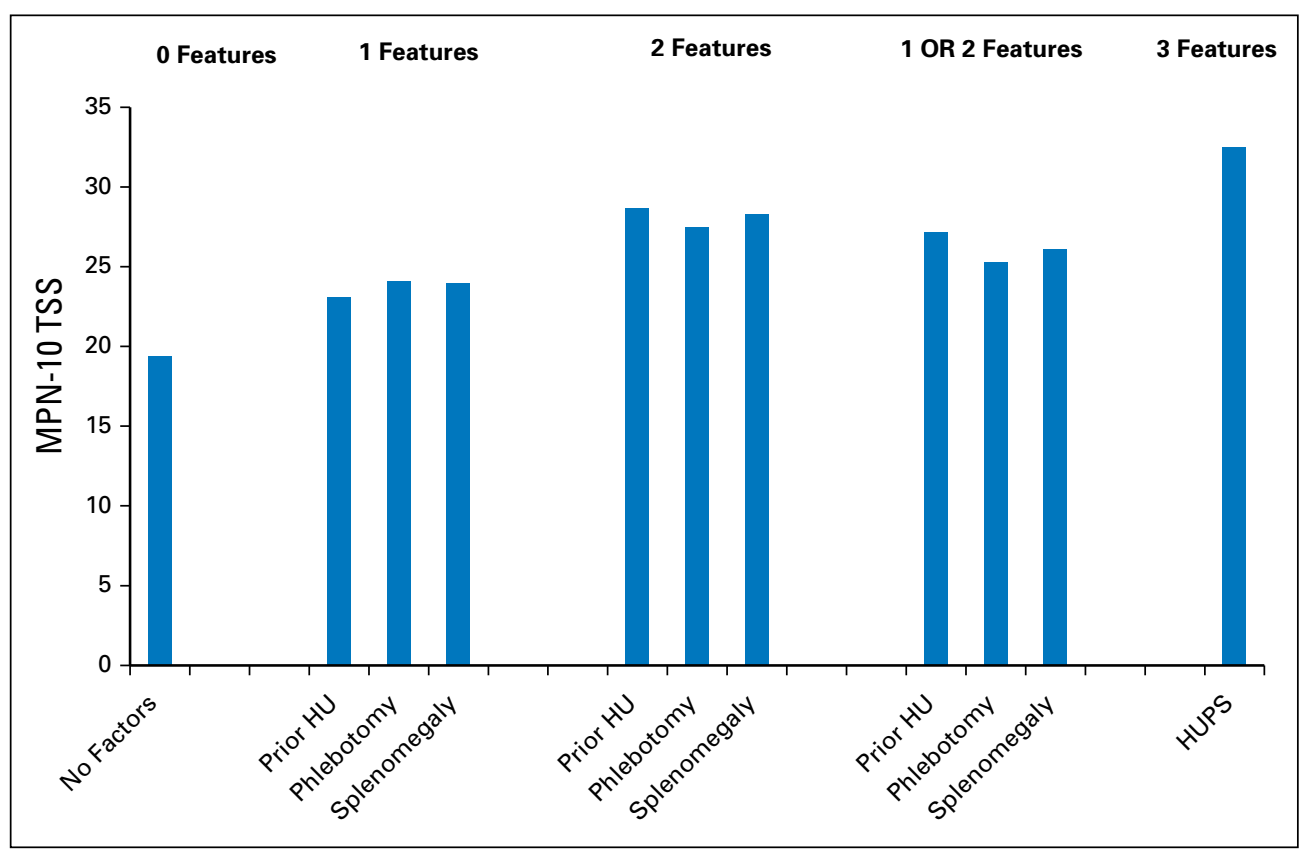

Fig 4. Myeloproliferative neoplasm symptom assessment form (MPN-10) total symptom score (TSS) according to the number of features. HU, hydroxyurea; HUPS, all three features (known HU use, known phlebotomy, and enlarged spleen).

\section{AUTHOR CONTRIBUTIONS}

Conception and design: Holly Geyer, Amylou C. Dueck, Zhenya Seyak, Ruben A. Mesa, Robyn Scherber

Provision of study materials or patients: Lydia Roy, Zhenya Seyak Collection and assembly of data: Holly Geyer, Amylou C. Dueck, Stefanie Slot, Kostanze Dohner, Karin Bonatz, Andreas Reiter, Francoise Boyer, Jean-Christophe Ianotto, Thomas Lehmann, Ruben A. Mesa Data analysis and interpretation: Holly Geyer, Robyn Scherber, Heidi Kosiorek, Amylou C. Dueck, Jean-Jacques Kiladjian, Zhijian Xiao, Sonja Zweegman, Federico Sackmann, Ana Kerguelen Fuentes, Dolores

\section{REFERENCES}

1. Tefferi A, Vardiman JW: Classification and diagnosis of myeloproliferative neoplasms: The 2008 World Health Organization criteria and point-of-care diagnostic algorithms. Leukemia 22:14-22, 2008

2. Emanuel RM, Dueck $A C$, Geyer $H L$, et al: Myeloproliferative neoplasm (MPN) symptom assessment form total symptom score: Prospective international assessment of an abbreviated symptom burden scoring system among patients with MPNs. J Clin Oncol 30:4098-4103, 2012

3. Mesa RA, Niblack J, Wadleigh $M$, et al: The burden of fatigue and quality of life in myeloproliferative disorders (MPDs): An international Internetbased survey of 1179 MPD patients. Cancer 109: 68-76, 2007

4. Berlin NI: Diagnosis and classification of the polycythemias. Semin Hematol 12:339-351, 1975

5. Anger BR, Seifried E, Scheppach J, et al: Budd-Chiari syndrome and thrombosis of other abdominal vessels in the chronic myeloproliferative diseases. Klin Wochenschr 67:818-825, 1989

6. Tefferi A, Rumi E, Finazzi G, et al: Survival and prognosis among 1545 patients with contemporary polycythemia vera: An international study. Leukemia 27:1874-1881, 2013

7. Emanuel R, Marchioli R, Dueck A, et al: Impact of hematocrit on symptom burden among polycythemia vera patients. European Hematology Association Annual Meeting Abstracts. 276, 2013

8. Marchioli R, Finazzi G, Specchia G, et al: CYTO-PV Collaborative Group: Cardiovascular events and intensity of treatment in polycythemia vera. $\mathrm{N}$ Engl J Med 368:22-33, 2013

9. Kiladjian JJ, Chevret $S$, Dosquet $C$, et al: Treatment of polycythemia vera with hydroxyurea and pipobroman: Final results of a randomized trial initiated in 1980. J Clin Oncol 29:3907-3913, 2011

10. Alvarez-Larrán $A$, Pereira $A$, Cervantes $F$, et al: Assessment and prognostic value of the European LeukemiaNet criteria for clinicohematologic response, resistance, and intolerance to hydroxyurea in polycythemia vera. Blood 119 : 1363-1369, 2012

11. Barosi G, Birgegard G, Finazzi G, et al: A unified definition of clinical resistance and intolerance to hydroxycarbamide in polycythaemia vera and primary myelofibrosis: Results of a European LeukemiaNet (ELN) consensus process. $\mathrm{Br} \mathrm{J}$ Haematol 148: 961-963, 2010
12. Vannucchi AM, Kiladjian JJ, Griesshammer M, et al: Ruxolitinib versus standard therapy for the treatment of polycythemia vera. N Engl J Med 372: 426-435, 2015

13. Scherber R, Dueck $A C$, Johansson $P$, et al: The myeloproliferative neoplasm symptom assessment form (MPN-SAF): International prospective validation and reliability trial in 402 patients. Blood 118:401-408, 2011

14. Scherber RM, Senyak $Z$, Dueck $A C$, et al: The impact of medical comorbidities in MPN-related fatigue. Blood 124:1862, 2014

15. Emanuel $R$, Dueck $A C$, Geyer $H L$, et al: Myeloproliferative (MPN) symptom burden response thresholds: Assessment of MPN-SAF TSS quartiles as potential markers of symptom response. Blood 122:4067, 2013

16. Vaidya R, Gangat $N$, Jimma $T$, et al: Plasma cytokines in polycythemia vera: phenotypic correlates, prognostic relevance, and comparison with myelofibrosis. Am J Hematol 87:1003-1005, 2012

17. Geyer $H L$, Scherber RM, Dueck $A C$, et al: Distinct clustering of symptomatic burden among myeloproliferative neoplasm patients: Retrospective assessment in 1,470 patients. Blood 123:3803-3810, 2014 


\section{Affiliations}

Holly Geyer, Robyn Scherber, Heidi Kosiorek, Amylou C. Dueck, and Ruben A. Mesa, Mayo Clinic, Scottsdale, AZ; Jean-Jacques Kiladjian, Hospital Saint-Louis, Paris; Francoise Boyer, Centre Hospitalier Universitaire, Angers; Gabriel Etienne, Institut Bergonie, Bordeaux; Jean-Christophe Ianotto, Centre Hospitalier Universitaire, Brest; Dana Ranta, Centre Hospitalier Universitaire, Grenoble; Lydia Roy, Centre Hospitalier Universitaire, Poitiers; Jean-Yves Cahn, Centre Hospitalier Universitaire, Grenoble, France; Zhijian Xiao, Zefeng $\mathrm{Xu}$, Yue Zhang, Xiujuan Sun, Junqing Xu, and Peihong Zhang, Chinese Academy of Medical Sciences and Peking Union Medical College, Tianjin, China; Stefanie Slot and Sonja Zweegman, VU University Medical Center, Amsterdam; Peter A.W. te Boekhorst, Erasmus Medical Center, Rotterdam; Suzan Commandeur, Leiden University Medical Center, Leiden; Harry Schouten, Maastricht University Medical Center, Maastricht, the Netherlands; Frederico Sackmann, Fundaleu, Buenos Aires, Argentina; Ana Kerguelen Fuentes and Dolores Hernández-Maraver, University Hospital La Paz, Madrid; Carlos Besses, Hospital del Mar; Francisco Cervantes, University of Barcelona, Barcelona, Spain; Konstanze Döhner and Frank Stegelmann, University Hospital of Ulm; Karin Bonatz and Andreas Reiter, Universitätsmedizin Mannheim, Mannheim; Heike L. Pahl, University Hospital Freiburg, Freiburg; Martin Griesshammer, Johannes Wesling Klinikum Minden, Germany; Claire N. Harrison and Deepti Radia, Guy's and St Thomas NHS Foundation Trust; Robert Peter Gale, Imperial College, London, United Kingdom; Pablo Muxi, Hospital Británico, Montevideo, Uruguay; Peter L. Johansson and Bjorn Andreasson, NU Hospital Organization, Uddevalla; Gunnar Birgegard, University Hospital, Uppsala; Jan Samuelsson, Stockholm South Hospital, Stockholm, Sweden; Alessandro Rambaldi, Tiziano Barbui, and Maria L. Ferrari, Ospedali Riuniti di Bergamo, Bergamo; Giovanni Barosi, IRCCS Policlinico S. Matteo Foundation; Francesco Passamonti, Fondazione IRCCS Policlinico San Matteo, University of Pavia, Pavia; Alessandro M. Vannucchi, Ospedale di Circolo, Varese, Italy; Norman Maldonado, University of Puerto Rico School of Medicine, San Juan, Puerto Rico; Thomas Lehmann, University Hospital Basel, Basel, Switzerland; and Zhenya Senyak, MPN Forum, Asheville, NC 


\section{AUTHORS' DISCLOSURES OF POTENTIAL CONFLICTS OF INTEREST}

Symptomatic Profiles of Patients With Polycythemia Vera: Implications of Inadequately Controlled Disease

The following represents disclosure information provided by authors of this manuscript. All relationships are considered compensated. Relationships are self-held unless noted. I = Immediate Family Member, Inst = My Institution. Relationships may not relate to the subject matter of this manuscript. For more information about ASCO's conflict of interest policy, please refer to www.asco.org/rwc or jco.ascopubs.org/site/ifc.

Holly Geyer

No relationship to disclose

\section{Robyn Scherber}

No relationship to disclose

Heidi Kosiorek

No relationship to disclose

Amylou C. Dueck

No relationship to disclose

Jean-Jacques Kiladjian

Honoraria: Novartis, Shire

Consulting or Advisory Role: Novartis, Shire, Incyte

Research Funding: AOP Orphan Pharmaceuticals (Inst), Novartis (Inst)

Travel, Accommodations, Expenses: Novartis, Incyte

Zhijian Xiao

No relationship to disclose

Stefanie Slot

Consulting or Advisory Role: Novartis

Research Funding: Novartis (Inst)

Travel, Accommodations, Expenses: Novartis

Sonja Zweegman

Research Funding: Novartis (Inst)

Federico Sackmann

No relationship to disclose

Ana Kerguelen Fuentes

No relationship to disclose

Dolores Hernandez-Maraver

No relationship to disclose

Kostanze Dohner

No relationship to disclose

Claire N. Harrison

Speakers' Bureau: CTI, Sanofi, Novartis, Shire

Research Funding: Novartis

Deepti Radia

No relationship to disclose

Pablo Muxi

Travel, Accommodations, Expenses: Novartis

Carlos Besses

No relationship to disclose

Francisco Cervantes

No relationship to disclose

Peter L. Johansson

No relationship to disclose

Bjorn Andreasson

No relationship to disclose

Alessandro Rambaldi

Consulting or Advisory Role: Pierre Fabre Pharma

Travel, Accommodations, Expenses: Pierre Fabre Pharma
Tiziano Barbui

Speakers' Bureau: Novartis

Travel, Accommodations, Expenses: Novartis

Karin Bonatz

No relationship to disclose

Andreas Reiter

Honoraria: Novartis Pharma

Consulting or Advisory Role: Novartis Pharma

Travel, Accommodations, Expenses: Novartis Pharma

Francoise Boyer

No relationship to disclose

Gabriel Etienne

Honoraria: Novartis

Consulting or Advisory Role: Novartis

Speakers' Bureau: Novartis

\section{Jean-Christophe Ianotto}

Consulting or Advisory Role: Deux communications in French local reunions

Travel, Accommodations, Expenses: Novartis

Dana Ranta

Consulting or Advisory Role: Novartis

Travel, Accommodations, Expenses: Sandoz

Lydia Roy

Consulting or Advisory Role: Novartis, Bristol-Meyers Squibb

Speakers' Bureau: Novartis, Shire

Research Funding: Bristol-Meyers Squibb (Inst), Merck (Inst)

Travel, Accommodations, Expenses: Novartis, Bristol-Meyers Squibb

Jean-Yves Cahn

No relationship to disclose

Norman Maldonado

No relationship to disclose

Giovanni Barosi

No relationship to disclose

Maria L. Ferrari

No relationship to disclose

Robert Peter Gale

No relationship to disclose

Gunnar Birgegard

Consulting or Advisory Role: Shire Pharmaceuticals

Travel, Accommodations, Expenses: Shire Pharmaceuticals, Vifor

Zefeng Xu

No relationship to disclose

Yue Zhang

No relationship to disclose

Xiujuan Sun

No relationship to disclose

Junqing Xu

No relationship to disclose 


\section{Peihong Zhang}

No relationship to disclose

Peter A.W. te Boekhorst

Consulting or Advisory Role: Novartis, CTI, Alexion Pharmaceuticals Travel, Accommodations, Expenses: Novartis, CTI, Alexion

Pharmaceuticals

\section{Suzan Commandeur}

Employment: Johnson \& Johnson

Honoraria: Johnson \& Johnson

\section{Harry Schouten}

No relationship to disclose

\section{Heike L. Pahl}

Honoraria: Novartis, AOP Pharma

Consulting or Advisory Role: Novartis

Speakers' Bureau: Novartis

Research Funding: Novartis

Patents, Royalties, Other Intellectual Property: Patent on PRV-1

\section{Martin Griesshammer}

No relationship to disclose

\section{Frank Stegelmann}

No relationship to disclose
Thomas Lehmann

Consulting or Advisory Role: Novartis, Roche, Gilead Sciences Research Funding: Novartis

Travel, Accommodations, Expenses: Novartis, Roche, Bristol-Meyers Squibb

Zhenya Senyak

No relationship to disclose

Alessandro M. Vannucchi

Consulting or Advisory Role: Novartis

Speakers' Bureau: Novartis

Research Funding: Novartis

Francesco Passamonti

No relationship to disclose

Jan Samuelsson

Consulting or Advisory Role: Novartis Sweden

Ruben A. Mesa

Honoraria: Novartis

Consulting or Advisory Role: Novartis

Research Funding: Incyte (Inst), Gilead Sciences (Inst), CTI (Inst), Promedior (Inst) 